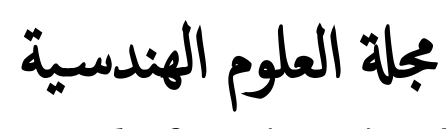

FES Journal of Engineering Sciences

http://journal.oiu.edu.sd/ojs/index.php/fjes/index

\title{
Homotopy Perturbation Transform Method for Extensible Beam Equations
}

\author{
Khaled A. Ishag ${ }^{1, *}$ and Faris A. Okasha ${ }^{1}$ \\ 1 Department of Basic Sciences, Omdurman Islamic University, Omdurman, Sudan. \\ *Corresponding author: Khaled A. Ishag (e-mail: khalid.a.ishag@oiu.edu.sd ). \\ Article history: Received 06 March 2019, Received in revised form 05 May 2019, Accepted 16 May 2019
}

\begin{abstract}
In this paper, we apply analytical method (homotopy perturbation transform method), for solving nonlinear partial differential equation of extensible beam, we discuss three partial differential equations generating from model. The first equation is called longitudinal vibrations of a beam, second equation is called transverse vibrations of a beam and third equation is called extensible beam. The equation of extensible beam was defined by Woiniwsky- Krieger as a model for transverse deflection of an extensible beam of natural length whose ends are held a fixed distance apart.
\end{abstract}

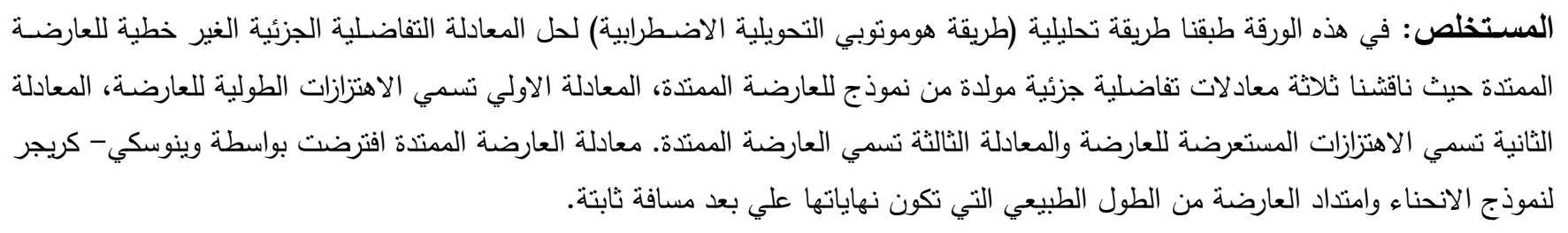

Keywords: partial differential equations, homotopy perturbation method, Laplace's Transforms, MATLAB programming.

\section{INTRODUCTION}

Beams are the most common type of structural component, particularly in Civil and Mechanical Engineering. A beam is a bar-like structural member whose primary function is to support transverse loading and carry it to the supports, this equation describes the motion of a beam initially located on the $x$-axis which is vibrating transversely "perpendicular to the $x$-direction", in this case $u(x, t)$ is the transverse displacement or deflection at any time $t$ of any points $x$ [1].

In this paper we using homotopy perturbation transform method and representation graphic of the solutions by using MATLAB program to solving extensible beam linear or/and nonlinear partial differential equation dependent for parameter $\gamma$, by using. This method is applied in a direct way without using linearization, transformation, discretization or restrictive assumptions. The method gives more realistic series solutions that converge very rapidly in engineering problems. The fact that the homotopy perturbation transform method solves nonlinear problems without using the Adomian's polynomials. The extensible beam equation denoted by

$$
\begin{aligned}
& \frac{\partial^{2} u}{\partial t^{2}}+\alpha \frac{\partial^{4} u}{\partial x^{4}}-(\beta \\
& \left.+\gamma \int_{0}^{L}\left(u_{\xi}(\xi, t)\right)^{2} d \xi\right) \frac{\partial^{2} u}{\partial x^{2}}=0
\end{aligned}
$$

where the constants $\alpha$ and $\gamma$ are positive, $\alpha=E I /$ $\rho, \beta=E A / \rho L$ and $\gamma=E A / 2 \rho L$, where $E$ is young's modulus, $I$ is the cross sectional of second moment area, $\rho$ is density and $A$ is the cross sectional area.

Equation (1) was proposed by Woiniwsky- Krieger as a model for transverse deflection of an extensible beam of natural length whose ends are held a fixed distance apart [2, 3]. If

i. $\alpha=0 \& \gamma=0$, Eq. (1) is called longitudinal vibrations of a beam. 
ii. $\beta=0 \& \gamma=0$, Eq. (1) is called transverse vibrations of a beam.

iii. $\gamma=0$, Eq. (1) is linear partial differential equation.

Consider the initial- boundary value problem at both ends.

BC $u(0, t)=u(L, t)=u_{x}(0, t)=u_{x}(L, t)=0$

IC $u(x, 0)=f(x) \quad \& \quad u_{t}(x, 0)=g(x)$

\section{HOMOTOPY PERTURBATION METHOD (HPM)}

The homotopy perturbation method (HPM) was first proposed by He J. Huan in 1999, where the solution of this method is considered as the sum of an infinite series which is very rapidly converge to the accurate solution. Consider a general equation of type [4].

$$
L u+N u=\mathrm{g}(\mathrm{x}, \mathrm{t}), x \in \Omega
$$

where $L$ is linear operator, $N$ is nonlinear operator and $\mathrm{g}$ is known analytical function. We define a convex homotopy $H(u, p)$

by

$$
H(u, p): \Omega \times[0,1] \rightarrow R
$$

$$
H(u, p)=(1-p) F(u)+p(L u+N u-g)=0
$$

We have

$$
\begin{aligned}
& H(u, 0)=F(u) \& \\
& H(u, 1)=L u+N u-g
\end{aligned}
$$

The embedding parameter monotonically increases from zero to unity as the trivial problem $F(u)=0$, continuously deforms the original problem $L u=0$, the embedding parameter $p \in[0,1]$, can be considered as expanding parameter. The homotopy perturbation method use homotopy parameter $p$ as an expanding parameter to obtain.

$$
u(x, t)=\sum_{n=0}^{\infty} p^{n} u_{n}(x, t)
$$

If $p \rightarrow 1$, then Eq. (3) corresponds to Eq. (2), and became the approximate solution of the form

$$
\begin{gathered}
u(x, t)=\lim _{p \rightarrow 1} \sum_{n=0}^{\infty} p^{n} u_{n}(x, t)=\sum_{n=0}^{\infty} u_{n}(x, t) \\
=u_{0}+u_{1}+u_{2}+\cdots
\end{gathered}
$$

\subsection{Homotopy Perturbation Transform Method} (HPTM)

In this method, we using Laplace's transform of Eq. (3) and we suppose $F(u)$ is linear operator subset form original Eq. (2), we obtain

$$
\begin{gathered}
\mathcal{L}(L(u))+p \mathcal{L}((N u-\mathrm{g}))=\mathcal{L}(0) \\
u(x, s)=\frac{1}{s^{n}} \sum_{k=0}^{n-1} s^{(k+n-1)} \frac{\partial^{k} u(x, 0)}{\partial t^{k}} \\
-p \frac{1}{s^{n}} \mathcal{L}((N u-\mathrm{g}))
\end{gathered}
$$

Finally, we use Laplace's inverse

$$
\begin{aligned}
u(x, t)= & \mathcal{L}^{-1}\left(\frac{1}{s^{n}} \sum_{k=0}^{n-1} s^{(k+n-1)} \frac{\partial^{k} u(x, 0)}{\partial t^{k}}\right. \\
& \left.-p \frac{1}{s^{n}} \mathcal{L}((N u-g))\right)
\end{aligned}
$$

$$
\begin{aligned}
& \text { Put } \begin{array}{c}
u(x, t)=\sum_{n=0}^{\infty} p^{n} u_{n}(x, t) \\
\sum_{n=0}^{\infty} p^{n} u_{n}=\mathcal{L}^{-1}\left(\frac{1}{s^{n}} \sum_{k=0}^{n-1} s^{(k+n-1)} \frac{\partial^{k} u(x, 0)}{\partial t^{k}}\right. \\
\left.-p \frac{1}{s^{n}} \mathcal{L}\left(\left(N \sum_{n=0}^{\infty} p^{n} u_{n}-\mathrm{g}\right)\right)\right)
\end{array}
\end{aligned}
$$

Example 1: Solve string vibrating beam equation

$$
\frac{\partial^{2} u}{\partial t^{2}}-\beta \frac{\partial^{2} u}{\partial x^{2}}=0
$$

with initial conditions:

$$
u(x, 0)=\sin (x) \quad \& \quad u_{t}(x, 0)=0
$$

Using the Homotopy Perturbation Transform Method (HPTM), we have

$$
\begin{gathered}
(1-p) \frac{\partial^{2} u}{\partial t^{2}}+p\left(\frac{\partial^{2} u}{\partial t^{2}}-\beta \frac{\partial^{2} u}{\partial x^{2}}\right)=0 \\
\frac{\partial^{2} u}{\partial t^{2}}=p \beta \frac{\partial^{2} u}{\partial x^{2}}
\end{gathered}
$$

Using Laplace's Transform of both sides

$$
\begin{gathered}
s^{2} u(x, s)-s u(x, 0)-u_{t}(x, 0)=p \beta \mathcal{L}\left(\frac{\partial^{2} u}{\partial x^{2}}\right) \\
u(x, s)=\frac{u(x, 0)}{s}+\frac{u_{t}(x, 0)}{s^{2}}+p \beta\left(\frac{1}{s^{2}} \mathcal{L}\left(\frac{\partial^{2} u}{\partial x^{2}}\right)\right)
\end{gathered}
$$

Given $u(x, 0)=\sinh (x) \quad \& \quad u_{t}(x, 0)=0$ and let $\beta=1$, so

$$
u(x, t)=\sinh (x)+p \mathcal{L}^{-1}\left(\frac{1}{s^{2}} \mathcal{L}\left(\frac{\partial^{2} u}{\partial x^{2}}\right)\right)
$$

Put $\quad u=\sum_{n=0}^{\infty} p^{n} u_{n}$ 


$$
\begin{aligned}
& \sum_{n=0}^{\infty} p^{n} u_{n} \\
& =\sinh (x) \\
& +p \mathcal{L}^{-1}\left(\frac{1}{s^{2}} \mathcal{L}\left(\frac{\partial^{2}\left(\sum_{n=0}^{\infty} p^{n} u_{n}(x, t)\right)}{\partial x^{2}}\right)\right)
\end{aligned}
$$

Compare the coefficients of power of $p$

$$
\begin{array}{cc}
p^{0}: & u_{0}=\sinh (x) \\
p^{1}: & u_{1}=\mathcal{L}^{-1}\left(\frac{1}{s^{2}} \mathcal{L}\left(\frac{\partial^{2}}{\partial x^{2}}\left(u_{0}\right)\right)\right) \\
u_{1}=\mathcal{L}^{-1}\left(\frac{\sinh (x)}{s^{3}}\right)=-\sinh (x) \frac{t^{2}}{2 !} \\
p^{2}: \quad u_{2}=\mathcal{L}^{-1}\left(\frac{1}{s^{2}} \mathcal{L}\left(\frac{\partial^{2}}{\partial x^{2}}\left(u_{1}\right)\right)\right) \\
u_{2}=\mathcal{L}^{-1}\left(\frac{\sinh (x)}{s^{5}}\right)=\sinh (x) \frac{t^{4}}{4 !} \\
p^{3}: \quad u_{3}=\mathcal{L}^{-1}\left(\frac{1}{s^{2}} \mathcal{L}\left(\frac{\partial^{2}}{\partial x^{2}}\left(u_{2}\right)\right)\right) \\
u_{3}=\mathcal{L}^{-1}\left(\frac{\sinh (x)}{s^{7}}\right)=\sinh (x) \frac{t^{6}}{6 !}
\end{array}
$$$$
p^{n}: \quad u_{n}=\mathcal{L}^{-1}\left(\frac{1}{s^{2}} \mathcal{L}\left(\frac{\partial^{2}}{\partial x^{2}}\left(u_{n-1}\right)\right)\right)
$$$$
u_{n}=\sin h(x) \frac{t^{2 n}}{(2 n) !}
$$$$
\begin{gathered}
u(x, t)=\sum_{\substack{k=0 \\
u}}^{\infty} u_{k}(x, t)=\sum_{k=0}^{\infty} \sinh (x) \frac{t^{2 k}}{(2 k) !} \\
u(x, t)=\sinh (x) \cosh (t)
\end{gathered}
$$

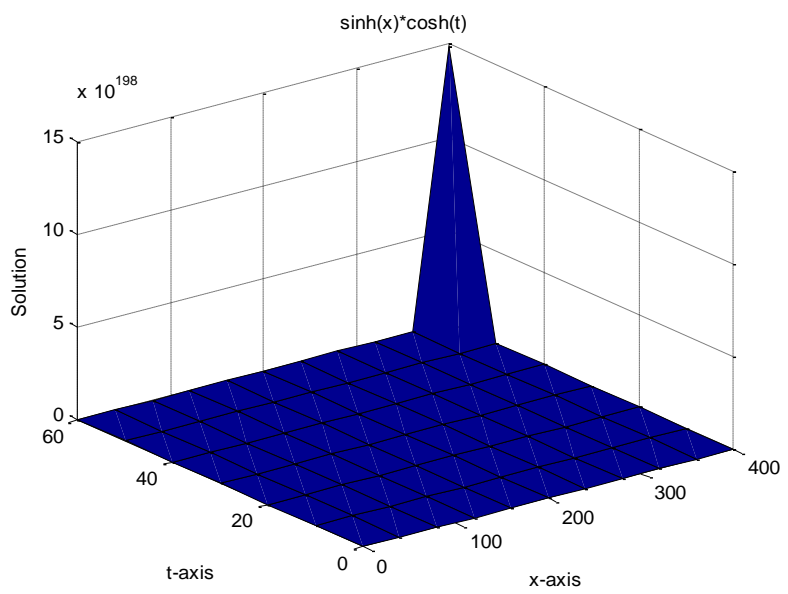

Fig. 1: Graphical Representation of Example 2
Example 2: Solve transverse vibrating beam equation

$$
\frac{\partial^{2} u}{\partial t^{2}}+\alpha \frac{\partial^{4} u}{\partial x^{4}}=0
$$

with initial conditions:

$$
u(x, 0)=\sin (x) \quad \& \quad u_{t}(x, 0)=x^{2}
$$

Using the Homotopy Perturbation Transform Method (HPTM), we have

$$
\begin{gathered}
(1-p) \frac{\partial^{2} u}{\partial t^{2}}+p\left(\frac{\partial^{2} u}{\partial t^{2}}+\alpha \frac{\partial^{4} u}{\partial x^{4}}\right)=0 \\
\frac{\partial^{2} u}{\partial t^{2}}=-\alpha p \frac{\partial^{4} u}{\partial x^{4}}
\end{gathered}
$$

Using Laplace's Transform of both sides

$$
\begin{aligned}
& s^{2} u(x, s)-s u(x, 0)-u_{t}(x, 0)=-\alpha p \mathcal{L}\left(\frac{\partial^{4} u}{\partial x^{4}}\right) \\
& u(x, s)=\frac{u(x, 0)}{s}+\frac{u_{t}(x, 0)}{s^{2}}-\alpha p\left(\frac{1}{s^{2}} \mathcal{L}\left(\frac{\partial^{4} u}{\partial x^{4}}\right)\right)
\end{aligned}
$$

Given $u(x, 0)=\sin (x) \quad \& \quad u_{t}(x, 0)=x^{2}$ and let $\alpha=-1$, so

$$
\begin{aligned}
& \begin{array}{l}
u(x, t)=\sin (x)+x^{2} t+p \mathcal{L}^{-1}\left(\frac{1}{s^{2}} \mathcal{L}\left(\frac{\partial^{4} u}{\partial x^{4}}\right)\right) \\
\text { Put } \quad u=\sum_{n=0}^{\infty} p^{n} u_{n} \\
\qquad \sum_{n=0}^{\infty} p^{n} u_{n} \\
\quad=\sin (x)+x^{2} t \\
+p \mathcal{L}^{-1}\left(\frac{1}{s^{2}} \mathcal{L}\left(\frac{\partial^{4}\left(\sum_{n=0}^{\infty} p^{n} u_{n}(x, t)\right)}{\partial x^{4}}\right)\right)
\end{array}
\end{aligned}
$$

Compare the coefficients of power of $p$

$$
\begin{array}{cc}
p^{0}: & u_{0}=\sin (x)+x^{2} t \\
p^{1}: & u_{1}=\mathcal{L}^{-1}\left(\frac{1}{s^{2}} \mathcal{L}\left(\frac{\partial^{4}}{\partial x^{4}}\left(u_{0}\right)\right)\right) \\
u_{1}=\mathcal{L}^{-1}\left(\frac{\sin (x)}{s^{3}}\right)=\sin (x) \frac{t^{2}}{2 !} & u_{2}=\mathcal{L}^{-1}\left(\frac{1}{s^{2}} \mathcal{L}\left(\frac{\partial^{4}}{\partial x^{4}}\left(u_{1}\right)\right)\right) \\
p^{2}: \quad u_{3}=\mathcal{L}^{-1}\left(\frac{1}{s^{2}} \mathcal{L}\left(\frac{\partial^{4}}{\partial x^{4}}\left(u_{1}\right)\right)\right) \\
u_{2}: \quad\left(\frac{\sin (x)}{s^{5}}\right)=\sin (x) \frac{t^{4}}{4 !} \\
u_{3}=\mathcal{L}^{-1}\left(\frac{\sin (x)}{s^{7}}\right)=\sin (x) \frac{t^{6}}{6 !}
\end{array}
$$




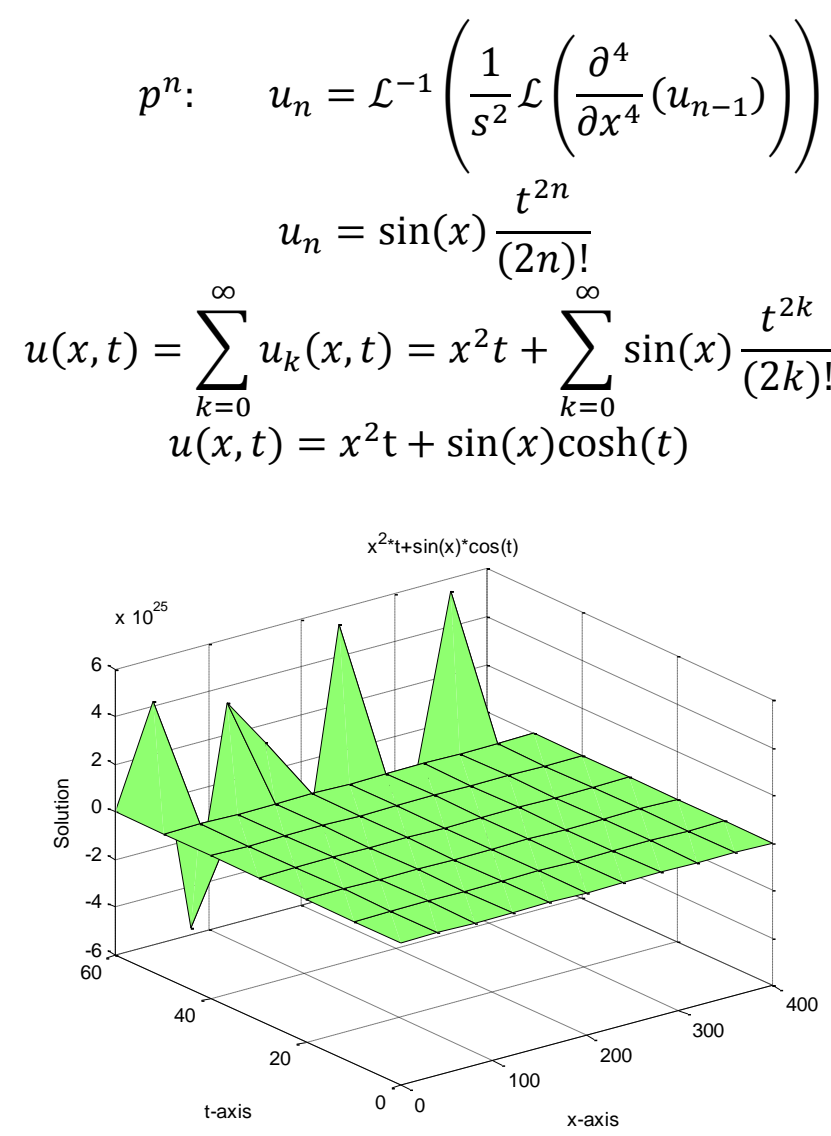

Fig. 2: Graphical Representation of Example 2

Example 2: Solve extensible beam equation

$$
\begin{aligned}
& \frac{\partial^{2} u}{\partial t^{2}}+\alpha \frac{\partial^{4} u}{\partial x^{4}}-(\beta \\
& \left.+\gamma \int_{0}^{L}\left(u_{\xi}(\xi, t)\right)^{2} d \xi\right) \frac{\partial^{2} u}{\partial x^{2}}=0
\end{aligned}
$$

with initial conditions:

$$
u(x, 0)=\frac{x^{2}}{2 !} \quad \& \quad u_{t}(x, 0)=0
$$

Using the Homotopy Perturbation Transform Method (HPTM) [5] , we have

$$
\begin{gathered}
(1-p) \frac{\partial^{2} u}{\partial t^{2}}+p\left(\frac{\partial^{2} u}{\partial t^{2}}+\alpha \frac{\partial^{4} u}{\partial x^{4}}-(\beta\right. \\
\left.\left.+\gamma \int_{0}^{L}\left(u_{\xi}(\xi, t)\right)^{2} d \xi\right) \frac{\partial^{2} u}{\partial x^{2}}\right)=0 \\
\frac{\partial^{2} u}{\partial t^{2}}=-p\left(\alpha \frac{\partial^{4} u}{\partial x^{4}}-(\beta\right. \\
\left.\left.+\gamma \int_{0}^{L}\left(u_{\xi}(\xi, t)\right)^{2} d \xi\right) \frac{\partial^{2} u}{\partial x^{2}}\right)
\end{gathered}
$$

Using Laplace's Transform of both sides

$$
\begin{aligned}
& s^{2} u(x, s)-s u(x, 0)-u_{t}(x, 0) \\
& =-p \mathcal{L}\left(\alpha \frac{\partial^{4} u}{\partial x^{4}}-(\beta\right. \\
& \left.\left.\quad+\gamma \int_{0}^{L}\left(u_{\xi}(\xi, t)\right)^{2} d \xi\right) \frac{\partial^{2} u}{\partial x^{2}}\right)
\end{aligned}
$$$$
u(x, s)=\frac{u(x, 0)}{s}+\frac{u_{t}(x, 0)}{s^{2}}-p \mathcal{L}\left(\alpha \frac{\partial^{4} u}{\partial x^{4}}-(\beta\right.
$$$$
\left.\left.+\gamma \int_{0}^{L}\left(u_{\xi}(\xi, t)\right)^{2} d \xi\right) \frac{\partial^{2} u}{\partial x^{2}}\right)
$$

Given $u(x, 0)=\frac{x^{2}}{2 !} \quad \& \quad u_{t}(x, 0)=0$, so

$$
\begin{aligned}
u(x, t)= & \frac{x^{2}}{2 !}-p \mathcal{L}^{-1}\left(\frac { 1 } { s ^ { 2 } } \mathcal { L } \left(\alpha \frac{\partial^{4} u}{\partial x^{4}}-(\beta\right.\right. \\
& \left.\left.\left.+\gamma \int_{0}^{L}\left(u_{\xi}(\xi, t)\right)^{2} d \xi\right) \frac{\partial^{2} u}{\partial x^{2}}\right)\right)
\end{aligned}
$$

Put $\quad u=\sum_{n=0}^{\infty} p^{n} u_{n}$

$\sum_{n=0}^{\infty} p^{n} u_{n}$

$$
=\frac{x^{2}}{2 !}
$$

$-p \mathcal{L}^{-1}\left(\frac{1}{s^{2}} \mathcal{L}\left(\alpha \frac{\partial^{4}\left(\sum_{n=0}^{\infty} p^{n} u_{n}(x, t)\right)}{\partial x^{4}}-(\beta\right.\right.$

$\left.\left.\left.+\gamma \int_{0}^{L}\left(\frac{\partial \sum_{n=0}^{\infty} p^{n} u_{n}(\xi, t)}{\partial \xi}\right)^{2} d \xi\right) \frac{\partial^{2} \sum_{n=0}^{\infty} p^{n} u_{n}(x, t)}{\partial x^{2}}\right)\right)$

Compare the coefficients of power of $p$

$$
\begin{array}{r}
p^{0}: \quad u_{0}=\frac{x^{2}}{2 !} \\
p^{1}: \quad u_{1}=-\mathcal{L}^{-1}\left(\frac { 1 } { s ^ { 2 } } \mathcal { L } \left(\alpha \frac{\partial^{4}}{\partial x^{4}}\left(u_{0}\right)-(\beta\right.\right. \\
\left.\left.\left.\left.+\gamma \int_{0}^{L}\left(\frac{\partial u_{0}(\xi, t)}{\partial \xi}\right)^{2} d \xi\right) \frac{\partial^{2} u_{0}}{\partial x^{2}}\right)\right)\right) \\
u_{1}=-\mathcal{L}^{-1}\left(\frac { 1 } { s ^ { 2 } } \mathcal { L } \left(\alpha \frac{\partial^{4}}{\partial x^{4}}\left(\frac{x^{2}}{2 !}\right)-(\beta\right.\right. \\
\left.\left.\left.\left.+\gamma \int_{0}^{L} \xi^{2} d \xi\right) \frac{\partial^{2}}{\partial x^{2}}\left(\frac{x^{2}}{2 !}\right)\right)\right)\right)
\end{array}
$$




$$
\begin{aligned}
u_{1}=-\mathcal{L}^{-1}\left(\frac{1}{s^{2}} \mathcal{L}\left(-\left(\beta+\gamma \int_{0}^{L} \xi^{2} d \xi\right)\right)\right) \\
u_{1}=\left(\beta+\gamma \frac{L^{4}}{12}\right) \frac{t^{2}}{2 !} \\
p^{2}: \quad u_{2}=-\mathcal{L}^{-1}\left(\frac{1}{s^{2}} \mathcal{L}\left(\frac{\partial^{4}}{\partial x^{4}}\left(u_{1}\right)\right)\right)
\end{aligned}
$$

$u_{2}$

$$
\begin{gathered}
=-\mathcal{L}^{-1}\left(\frac { 1 } { s ^ { 2 } } \mathcal { L } \left(\alpha \frac{\partial^{4}}{\partial x^{4}}\left(u_{1}\right)-(\beta\right.\right. \\
+\gamma \int_{0}^{L}\left(\left(\frac{\partial u_{0}(\xi, t)}{\partial \xi}\right)^{2} d \xi \frac{\partial^{2} u_{1}}{\partial x^{2}}\right. \\
\left.\left.\left.\left.+\int_{0}^{L} 2 \frac{\partial u_{0}(\xi, t)}{\partial \xi} \frac{\partial u_{1}(\xi, t)}{\partial \xi} d \xi\right) \frac{\partial^{2} u_{0}}{\partial x^{2}}\right)\right)\right) \\
u_{2}=-\mathcal{L}^{-1}\left(\frac{1}{s^{2}} \mathcal{L}(\alpha(0)-(\beta\right. \\
+\gamma \int_{0}^{L}\left(\xi^{2} d \xi(0)\right. \\
\left.\left.\left.\left.+\int_{0}^{L} 2 \xi(0) d \xi\right)(1)\right)\right)\right) \\
u_{2}=0
\end{gathered}
$$

$$
\begin{gathered}
u(x, t)=\sum_{k=0}^{\infty} u_{k}(x, t) \\
=u_{0}(x, t)+u_{1}(x, t)+u_{2}(x, t) \\
+\cdots \\
u(x, t)=\frac{x^{2}}{2 !}+\left(\beta+\gamma \frac{L^{4}}{12}\right) \frac{t^{2}}{2 !}
\end{gathered}
$$

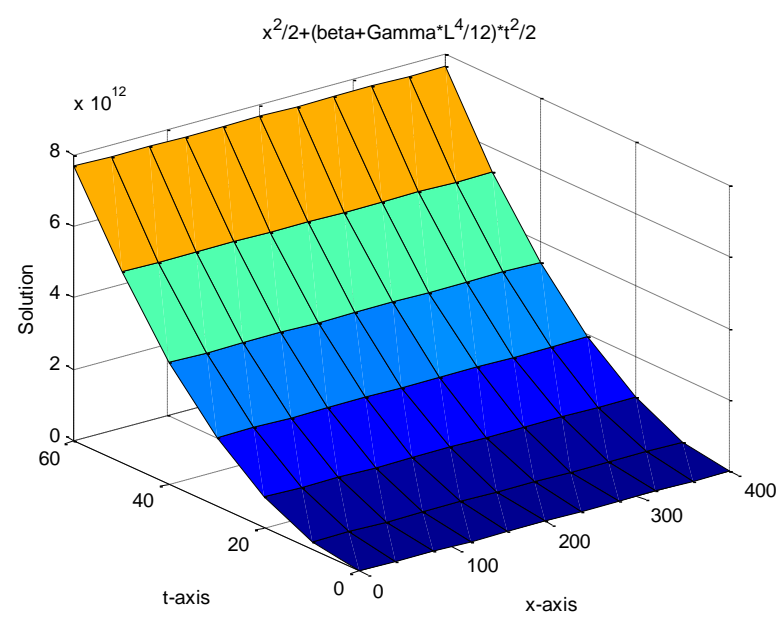

Fig. 3: Graphical Representation of Example 2, when $\beta=1 \& \gamma=2$

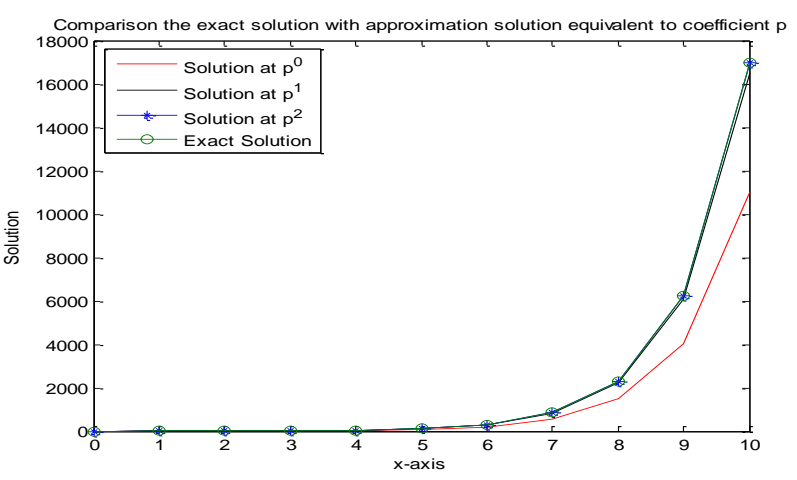

Fig. 4: Comparison the exact solution with approximation solution equivalent to coefficient $p$ for example 1

\section{Conclusions}

In this paper, we discuss the solutions of extensible beam linear or/and nonlinear partial differential equation dependent for parameter $\gamma$, by using homotopy perturbation transform method and representation graphic of the solutions by using MATLAB program. The method is applied in a direct way without using linearization, transformation, discretization or restrictive assumptions. The method gives more realistic series solutions that converge very rapidly in engineering problems. The fact that the HPTM solves nonlinear problems without using the Adomian's polynomials is a clear advantage of this technique over the decomposition method, in this method we only need the initial conditions of the application and choice the initial conditions are playing the convergence of the solution. 


\section{REFERENCES}

[1] Zienkiwicz, The finite element method fifth edition, volume 2 solid mechanics, 2000.

[2] J. M. Ball, Initial - Boundary value problems for an extensible beam, journal of mathematical analysis and applications, 1973, 42: $61-90$.

[3] He J. H, Homotopy Perturbation Technique, Comput. Math. Appl. Mech. Engrg, 1999, Vol.178, pp.257-262.

[4] Khaled A. Ishag, O. M. El Mekki and Emadeldeen A. Abdalrahim, Homotopy Perturbation method for Solving Transverse Vibrations of a Beam, Sudan Journal for Basic Sciences (SJBS),M 2019, 15: 1-11.

[5] Dennis G. Zill, Micheal R. Cullen, Differential equations with boundary value problems, 2009

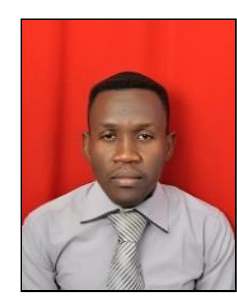

Khaled Abdalla Ishag Abdalla received his B.Sc. in Mathematics from Omdurman Islamic University, Sudan in 2011, M.Sc. in Industrial \& Computational Mathematics from Khartoum University, Khartoum, Sudan in 2014, $\mathrm{PhD}$ in Computational
Mathematics from Sudan University of Science and Technology, Khartoum, Sudan in 2019. He has been appointed as a lecturer at Faculty of Engineering Sciences, Omdurman Islamic University, Sudan, since 2014.

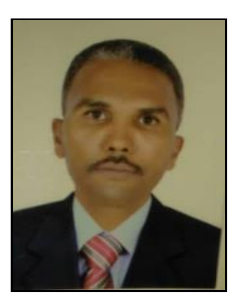

Faris Azhari Okasha Abdelgader received his B.Sc. in Mathematics - from Nile Valley University, Sudan in 2004, M.Sc. in Mathematics from Gezira University, Gezira, Sudan in 2007, PhD in Applied Mathematics from Sudan University of Science and Technology, Khartoum, Sudan in 2016. He has been appointed as a lecturer at Faculty of Engineering Sciences, Omdurman Islamic University, Sudan, since 2013. 